\title{
A New Method Combining Interior and Exterior Approaches for Linear Programming
}

\author{
Nguyen Ngoc Chu ${ }^{1}$, Pham Canh Duong ${ }^{2}$ and Le Thanh Hue ${ }^{3}$ \\ 1. Institute of Mathematics, 18 Hoang Quoc Viet, Hanoi, Vietnam. \\ 2. Center for Information Infrastructure Development, Vietnamese Academy of Science and Technology, 18 Hoang Quoc Viet, Hanoi,
} Vietnam.

3. Faculty of Informatics Technology, University of Mining and Geology, Hanoi, Vietnam.

Received: February 12, 2015 / Accepted: March 10, 2015 / Published: May 25, 2015.

\begin{abstract}
In this paper we present a new method combining interior and exterior approaches to solve linear programming problems. With the assumption that a feasible interior solution to the input system is known, this algorithm uses it and appropriate constraints of the system to construct a sequence of the so called station cones whose vertices tend very fast to the solution to be found. The computational experiments show that the number of iterations of the new algorithm is significantly smaller than that of the second phase of the simplex method. Additionally, when the number of variables and constraints of the problem increase, the number of iterations of the new algorithm increase in a slower manner than that of the simplex method.
\end{abstract}

Keywords: Linear programming, simplex method, station cone.

\section{Introduction}

After its discovery by Dantzig in 1947 [ 6 ] the simplex method was unrivaled, until the late 1980s, for its utility in solving practical linear programming problems. The computational experiments show that the simplex method is quite efficient in practice $[2,3,6,7]$. Nevertheless, there exists a class of linear programming problems for which the simplex method takes an exponential number of steps [10].

In 1979 [9] Khachian introduced the ellipsoid method which gives a bound of $O\left(n^{5} L\right)$ arithmetic operations on number with $O(n L)$ digits. Khachian's algorithm was of landmark importance for establishing the polynomial time solvability of linear programs. Despite its major theoretical advance, the ellipsoid method had little practical impact as the simplex method is more efficient for many classes of

Corresponding author: Nguyen Ngoc Chu, Ph.D., Research field: Operations Research, Economics. Institute of Mathematics, East Asia University of Technology. E-mail: dr.chu.vga@gmail.com. linear programming problems [1,14].

In 1984 [8] Kamarkar proposed a new projective method for linear programming problems which not only improved Khachian's theoretical worst-case polynomial bound but in fact promised dramatically practical performance improvement over simplex method. Karmarkar's algorithm requires $O\left(n^{3.5} L\right)$ operations on th $O(L)$ digit numbers as compared $\mathrm{f}$ $O\left(n^{6} L\right)$ such operations for the Khachian's ellipsoid method. Karmarkar's algorithm falls within the class of interior point methods. In contrast to the simplex method, which finds the optimal solution among the vertices of the feasible set, the interior point method moves through the interior of the feasible region and reaches the optimal solution only asymptotically. Stimulated by Karmarker's algorithm a variety of interior point methods were developed for linear programming $[12,16]$.

There are several important open problems in the theory of linear programming, the solution of which would represent fundamental breakthrough in 
mathematics. In the survey on linear programming [15] M.J. Todd has mentioned some unsolved problems: Is there a polynomial pivot rule for the simplex method? Does the bounded Hirsch conjecture hold? The immense efficiency of the simplex method in practice, despite its exponential time theoretical performance, hints that there may be variations of simplex algorithm that run in polynomial time.

In this paper we present a new interior exterior algorithm for solving linear programming problems. We assume that the linear programming problem has a initial strict interior point $O$. Then using this point $O$ we construct a sequence of the so called station cones whose vertices will allow a very fast optimal solution to be found.The new interior exterior algorithm has been tested, using MatLab, on a set of randomly generated linear problems. The computational experiments show that the number of iterations of the interior exterior approach is significantly smaller than that of the second phase of the simplex method.

The paper is organized as follows. In section 2 we introduce some results which are necessary for the construction of the algorithm. In section 3, we describe the criterion of selecting the leaving variables. The section 4 presents the main idea of the algorithm and proposes the selecting rule for entering vectors. The algorithm is presented in section 5. A numerical example has been illustrative in section 6 . The section 7 presents the computational experiments for some classes of small and medium size problems. Finally, some conclusions have been made in section 8 .

\section{Station Cone}

We consider a linear programming problem in the matrix form

$$
\begin{aligned}
& \max \langle c, x\rangle \\
& x \in P:=\{x \mid A x \leq b, x \geq 0\},
\end{aligned}
$$

where $\quad c \in \mathbb{R}^{n}, A \in A^{m \times n}, b \in \in \mathbb{R}^{m}, \forall x \in \mathbb{R}^{n}$. Let $A_{1}, A_{2}, \ldots, A_{m}$ denote the row vectors. Through this paper we suppose that (2.1) and its dual problem are nondegenerated .We also suggest the feasible region $P$ of (2.1) has strict interior points. For simplicity of argument, we assume that the matrix $A$ has full column rank $n$ and $n<m$.

Let $I_{n}=\left\{i_{1}, i_{2}, \ldots, i_{n}\right\} \subset\{1,2, \ldots, m\}$ such that the vectors $A_{i}, i \in I_{n}$ are linear independent. This means the vector $A_{i}, i \in I_{n}$ establish a basis of $R^{n}$. Therefore any vector $A_{l} \in \mathbb{R}^{n}$ can be expressed as a linear combination of the vectors $A_{i}, i \in I_{n}$. Let $\lambda_{l_{i_{k}}}$ be the linear coefficient of the vector $A_{l}$ in the basis $A_{i_{k}}, i_{k} \in I_{n}$, then

$$
a_{l j}=\sum_{k=1}^{n} \lambda_{l i_{k}} a_{i_{k} j}, j=1,2, \ldots, n, l=1,2, \ldots, m .
$$

Consider the system of homogeneous linear inequalities

$$
A_{i_{k}} x \leq 0, \quad i_{k} \in I_{n} .
$$

We indeed need to introduce the following definition.

Definition 1. The linear inequality

$$
A_{l} x \leq 0
$$

is called the consequent linear inequality of the system (2.2) if and only if all the solutions of the system (2.2) satisfy the linear inequality (2.3).

We need the following well known result in theory of linear inequalities.

Theorem 2.1 The linear inequality (2.3) is a consequent linear inequality of the system (2.2) if and only if

$$
A_{l}=\sum_{k=1}^{n} \lambda_{l_{k}} A_{i_{k}}, \lambda_{l_{i_{k}}} \geq 0, i_{k} \in I_{n}
$$

Definition 2. Let polyhedral cone $M$ be defined by system

$$
A_{i_{1}} x \leq b_{i_{1}}, A_{i_{2}} x \leq b_{i_{2}}, \ldots ., A_{i_{n}} x \leq b_{i_{n}},
$$

where $A_{i_{1}}, A_{i_{2}}, \ldots, A_{i_{n}}$ are linear independent. Then $M$ is called a station cone if the vector $c$ is a nonnegative linear combination of the vectors $A_{i_{1}}, A_{i_{2}}, \ldots, A_{i_{n}}$. The vertex $\bar{x}$ is called a station solution and the vectors $A_{i_{1}}, A_{i_{2}}, \ldots, A_{i_{n}}$ is called a 
basis of a station cone.

Therefore, geometrically it can be seen that all the station cones lie on one side of the objective function $(\mathrm{c}, \mathrm{x})$ at their vertices (see fig. 1: $M^{1}, M^{2}, M^{3}, M^{4}, M^{5}$ are station cones and $M^{6}, M^{7}, M^{8}, M^{9}$ are not station cones). In other words, the solutions of the system of linear inequalities that create the station cones satisfy the inequality $\langle c, x\rangle \leq\left\langle c, x^{*}\right\rangle$, whereas $x^{*}$ is the vertex of the station cones. This is equal to the fact that the inequality $\langle c, x\rangle \leq\left\langle c, x^{*}\right\rangle$ is the consequent inequality of the system of the linear inequalities, which formulate the station cone. This also means that the vector $\mathrm{c}$ is the nonnegative linear combination of the basic vectors of the station cone.

We have the following theorem

Theorem 2.2 If the station solution $\bar{x}$ satisfies all the constraints of the problem (2.1) then $\bar{x}$ is an optimal solution.

Proof. From the fact that $\bar{x}$ is a vertex of the station cone $M$, we have

$$
A_{i_{1}} \bar{x}=b_{i_{1}}, A_{i_{2}} \bar{x}=b_{i_{2}}, \ldots, A_{i_{n}} \bar{x}=b_{i_{n}} .
$$

Since $A_{i_{1}}, A_{i_{2}}, \ldots, A_{i_{n}}$ is a basis of a station cone then $c$ a nonnegative linear combination of $A_{i_{1}}, A_{i_{2}}, \ldots, A_{i_{n}}$. Therefore $\langle c, x\rangle \leq 0 \quad$ is a consequent inequality of the system

$$
A_{i_{1}} x \leq 0, A_{i_{2}} x \leq 0, \ldots, A_{i_{n}} x \leq 0 .
$$

This means that if $x^{1}$ is a solution of the system (2.4) then

$$
\left\langle c, x^{1}\right\rangle \leq 0
$$

From (2.5) and the fact that $\bar{x}$ is a vertex of $M$, it follows that

$$
\langle c, \bar{x}\rangle \geq\langle c, x\rangle, \quad \forall x \in M
$$

On the other hand

$$
\max \{\langle c, x\rangle \mid x \in M\} \geq \max \{\langle c, x\rangle \mid A x \leq b, x \geq 0\}
$$

Combine (2.6) and (2.7), we have

$$
\langle c, \bar{x}\rangle \geq \max \{\langle c, x\rangle \mid A x \leq b, x \geq 0\}
$$

If $\bar{x}$ satisfies all the constraints then (2.8) implies

$$
\langle c, \bar{x}\rangle=\max \{\langle c, x\rangle \mid A x \leq b, x \geq 0\} .
$$

This means $\bar{x}$ is an optimal solution of the problem (2.1). The proof is completed.

\section{Selecting the Leaving Vector}

Let $A_{i_{1}}, A_{i_{2}}, \ldots, A_{i_{n}}$ be the basis of the station cone and

$$
c=\sum_{k=1}^{n} \lambda_{k 0} A_{i_{k}}, A_{j}=\sum_{k=1}^{n} \lambda_{k j} A_{i_{k}}, \quad j=1,2, \ldots m
$$

Then from definion 2.1 follows that

$$
\lambda_{k o} \geq 0, \quad \forall k=1,2, \ldots n .
$$

From now on we assume that all $\lambda_{k o}$ are strictly positive, i.e.

$$
\lambda_{k 0}>0, k=1,2, \ldots, n \text {. }
$$

It is obvious that $\lambda_{k 0}>0, k=1,2, \ldots, n ; \lambda_{k 0}=0, k=n+1, \ldots, m$ is a basis solution of the dual problem of (2.1):

$$
\min \left\{\langle b, \lambda\rangle \backslash A^{T} \lambda \geq c^{T}, \lambda \geq 0\right\}
$$

where $\quad \lambda \in R^{m} \quad$. The assumption $\lambda_{k 0}>0, k=1,2, \ldots, n$ means that the dual problem (3.1) is nondegenerated.

Similarly to the simplex table, we establish the table of the coefficients $\lambda_{k j}$ in the following way:

\begin{tabular}{|l|l|l|l|l|l|l|l|l|}
\hline No & Basis & $c$ & $A_{1}$ & $A_{2}$ & $\ldots$ & $A_{s}$ & $\ldots$ & $A_{m}$ \\
\hline 1 & $A_{i_{1}}$ & $\lambda_{10}$ & $\lambda_{11}$ & $\lambda_{12}$ & $\ldots$ & $\lambda_{1 s}$ & $\ldots$ & $\lambda_{1 m}$ \\
\hline 2 & $A_{i_{2}}$ & $\lambda_{20}$ & $\lambda_{21}$ & $\lambda_{22}$ & $\ldots$ & $\lambda_{2 s}$ & $\ldots$ & $\lambda_{2 m}$ \\
\hline$\ldots$ & $\ldots$ & $\ldots$ & $\ldots$ & $\ldots$ & $\ldots$ & $\ldots$ & $\ldots$ & $\ldots$ \\
\hline$r$ & $A_{i_{r}}$ & $\lambda_{r 0}$ & $\lambda_{r 1}$ & $\lambda_{r 2}$ & $\ldots$ & $\lambda_{r s}$ & $\ldots$ & $\lambda_{r m}$ \\
\hline & & $\ldots$ & $\ldots$ & $\ldots$ & $\ldots$ & $\ldots$ & $\ldots$ & $\ldots$ \\
\hline$n$ & $A_{i_{n}}$ & $\lambda_{n 0}$ & $\lambda_{n 1}$ & $\lambda_{n 2}$ & & $\lambda_{n s}$ & $\ldots$ & $\lambda_{n m}$ \\
\hline
\end{tabular}




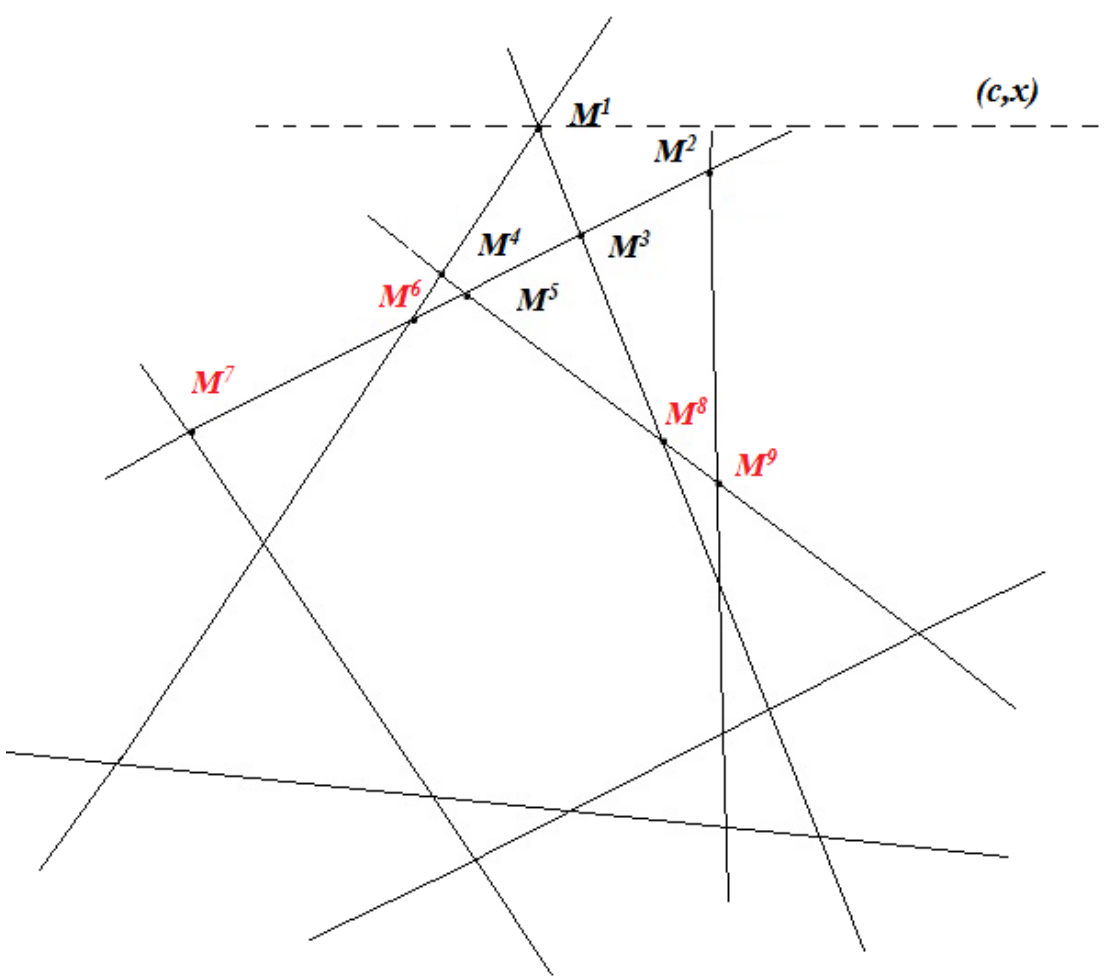

Fig. 1

Suppose $A_{\mathrm{i}_{\mathrm{r}}}$ is the leaving vector and $A_{s}$ is the entering vector. New coefficients $\lambda^{\prime}{ }_{k o}$ of vector $c$ can be calculated by the following formula

$$
\begin{aligned}
& \lambda_{r 0}^{\prime}=\frac{\lambda_{r 0}}{\lambda_{r s}}, \\
& \lambda_{k 0}^{\prime}=\lambda_{k 0}-\frac{\lambda_{r 0}}{\lambda_{r s}} \lambda_{k s}, \quad k \neq r, \\
& \frac{\lambda_{k 0}}{\lambda_{k s}} \geq \frac{\lambda_{r 0}}{\lambda_{r s}}, \quad \lambda_{r s}>0, \quad \lambda_{k s}>0 .
\end{aligned}
$$

From $\lambda_{k o}>0, \lambda^{\prime}{ }_{k o}>0, \forall k=1,2, \ldots n$ we follow that

$$
\begin{aligned}
& \lambda_{r 0}^{\prime}=\frac{\lambda_{r 0}}{\lambda_{r s}}>0, \\
& \lambda_{k 0}^{\prime}=\lambda_{k 0}-\frac{\lambda_{r 0}}{\lambda_{r s}} \lambda_{k s}, \quad k \neq r, \\
& \frac{\lambda_{k 0}}{\lambda_{k s}} \geq \frac{\lambda_{r 0}}{\lambda_{r s}}, \quad \lambda_{r s}>0, \quad \lambda_{k s}>0 .
\end{aligned}
$$

Therefore

$$
\frac{\lambda_{r 0}}{\lambda_{r s}}=\min _{k} \frac{\lambda_{k 0}}{\lambda_{k s}}, \lambda_{k s}>0, \lambda_{r s}>0
$$

The formulas (3.2), (3.3) guarantee that $A_{i_{1}}, A_{i_{2}}, \ldots, A_{i_{r-1}}, A_{s}, A_{i_{r+1}}, A_{i_{n}}$ are the basis of the station cone. So we have proved the following

Theorem 2.3 Let $A_{i_{1}}, A_{i_{2}}, \ldots, A_{i_{n}}$ be the basis of the station cone. Suppose we replaced $A_{i_{r}}$ by $A_{s}$. Then $A_{i_{1}}, \ldots, A_{i_{r-1}}, A_{s}, A_{i_{r+1}}, \ldots, A_{i_{n}}$ is the basis of the station cone if the leaving vector $A_{i_{r}}$ was chosen by condition

$$
\frac{\lambda_{r 0}}{\lambda_{r s}}=\min _{k} \frac{\lambda_{k 0}}{\lambda_{k s}}, \lambda_{k s}>0, \lambda_{r s}>0
$$

Now we have to show that formula (3.4) is hold.

Theorem 2.4 Among the coefficients $\lambda_{k s}, k=1$, $2, \ldots$, nat least one $\lambda_{r s}$ exists such that $\lambda_{r s}>0$

Proof. Suppose $\lambda_{k s} \leq 0, \forall k=1,2, \ldots, n$. Then the system

$$
A_{i} x \leq b_{i}, \quad i=i_{1}, i_{2}, \ldots, i_{n}, A_{s} x \leq b_{s}
$$

either would have a unique solution or would have no solution. This contradicts the assumption that the 
feasible set $P$ has interior points. So there exists at least one $\lambda_{r s}>0$. The theorem is proven $\square$

\section{Selecting the Entering Vector}

The idea of our algorithm is moving from one vertex $x^{k}$ of a station cone $M^{k}$ to another vertex $x^{k+1}$ of another station cone $M^{k+1}$ with a better value of the objective function. The movement depends on the cutting hyperplane $A_{s} x=b_{s}$ which will be defined by the intersection of the feasible polytope $P$ and the segment connecting the vertex $x^{k}$ of the station cone $M^{k}$ and the given interior point $O \in P$. The movement stops when the vertex $x^{k}$ of the station cone $M^{k}$ becomes a feasible point. The number of iterations will depend on the method of selecting the cutting hyperplane. We will illustrate the idea and the effectiveness of our algorithm by considering the following examples.

Let us approximate the equator of the earth by a polygon with the edge of 1 meter long. Then this polygon has 40 millions edges and 40 millions vertices. Suppose we have to find the maximum of a linear function $c x_{1}+c x_{2}$ over this polygon.

On figure 2 , let $A$ denote an optimal point, $B^{l}$ denote the starting point. Suppose the distance between $B^{l}$ and $A$ is 5 million meters. Then the simplex method will produce an optimal solution after 5 million iterations.

Let $M^{l}$ be a station cone defined by 2 constraints containing points $B^{l}$ and $D^{l}$, where $D^{l}$ is on the other side of $A$ with a distance, for examples, 4 million meters to $A$ (see fig.2).

We denote by $x^{1}$ the vertex of $M^{1}$. Since $M^{1}$ is a station cone, it is clear that

$$
\mathrm{cx}^{l} \geq c x, \forall x \in M^{l} .
$$

The station cone $M^{1}$ will be our starting cone. Starting our algorithm with the operation of connecting $x^{1}$ with $O$, where $O$ is the center of the equator. The segment $\left[x^{1}, 0\right]$ will intersect with the boundary of $P$ at $B^{2}$. Replacing the constraint containing $B^{1}$ by the constraint containing $B^{2}$ we have a new cone $M^{2}$. Repeat the above procedure with $M^{2}$ and we have $M^{3}$, etc. (see figure 2). The replacement of one constraint by another has to follow the restriction that the new generating cone is a station cone. We note that at each iteration, the distance between two points $B^{k}$ and $D^{k}$ defined by two edges of the station cone $M^{k}$ is reduced by approximately 2 times in comparison with the previous iteration. Therefore the number of the iterations $T$ can be estimated by the following bound

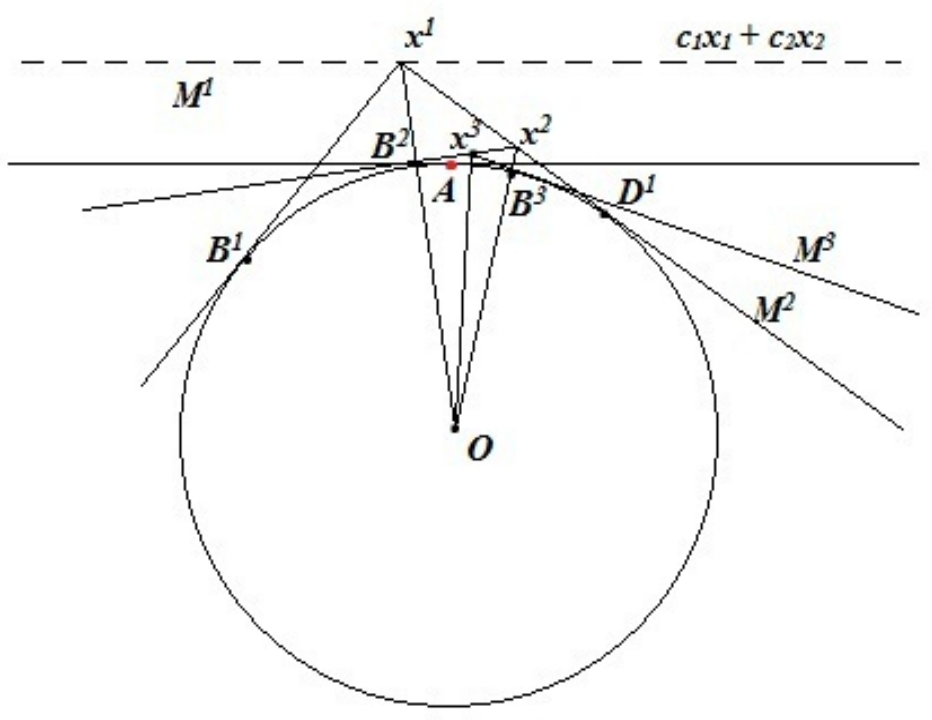

Fig. 2 


$$
T \approx \log _{2} \frac{m}{2}
$$

For our example with $m=40$ million the formula (4.1) gives

$$
T \approx \log _{2} \frac{m}{2}=\log _{2} 2.10^{7}<25
$$

The above example shows that our algorithm can produce an optimal solution after around 25 iterations.

We now proceed to find an initial station cone. We can find an initial station cone $M$ by solving the following system

$$
A^{T} \lambda=c^{T}, \lambda \geq 0,
$$

where $\lambda \in \mathbb{R}^{m}$. We can suppose $c^{T} \geq 0$ because, if some coefficient of $c^{T}$ is negative then we multiply both sides of the corresponding equation with -1 . To find a solution of (4.2), we solve the following big - $M$ problem

$$
\begin{array}{r}
\min \left\{M_{1} y_{1}+M_{2} y_{2}+\ldots+M_{n} y_{n}\right\} \\
A^{T} \lambda+E y=c^{T}, \lambda \geq 0, y \geq 0,
\end{array}
$$

Where, $\lambda \in \mathbb{R}^{m}, y \in \mathbb{R}^{n}$ and $E$ is the unit matrix of $(n \times n)$ and $M_{1}, M_{2}, \ldots, M_{n}$ are significantly large positive numbers. The problem (4.3) has an optimal solution $\lambda^{*} \geq 0, y^{*}=0$. and $\lambda^{*}$ is a solution of (4.2).

We also assume that a strict interior feasible solution $O$ of (2.1) is available. If such an initial point is not available then we modify the problem using the usual big - M augmentation [11] as follows:

$$
\begin{aligned}
& \max \left\{\langle c, x\rangle-M x_{n+1}\right\} \\
& A x-e x_{n+1} \leq b, x, x_{n+1} \geq 0 .
\end{aligned}
$$

Where $e=(1,1, \ldots, 1)^{T} \in \mathbb{R}^{m}$ and $M$ is a significantly large positive number.

Let $\quad x_{n+1}^{0}>\max \left\{0,-b_{1},-b_{2}, \ldots,-b_{m}\right\} \quad$.Then $\left(0, \ldots, 0, x_{n+1}^{0}\right)^{T}$ is a strict interior feasible solution of (4.4) which is in the same form as (2.1).

Let $O$ be a strict interior point of $P$. Denoted by $0^{i}, i=1,2, \ldots, n$ the projections of $O$ onto $n$ facets of the station cone $M^{k}$. Let $H_{i}, i=1,2, \ldots, n$ be the intersection points of the boundary of

$P$ and the segments $0,0^{i}, i=1,2, \ldots, n$. Then the new point $O^{*}$ will be calculated by the following formula

$$
O^{*}=\frac{1}{n+1}\left(\sum_{i=1}^{n} H_{i}+O\right)
$$

It is obvious that $O^{*}$ in (4.5) is the barycenter of the polytope $H_{1}, H_{2}, \ldots, H_{n}, 0$. Let us connect the point $O^{*}$ with vertex $x^{k}$ of the station cone $M^{k}$. Let $z^{k}$ denote the intersection point of $P$ and $\left[O^{*}, x^{k}\right]$, such that $z^{k} \in P,\left(z^{k}, x^{k}\right] \notin P$. Then the inequality $A_{s} x \leq b_{s}$ with $A_{s} z^{k}=b^{s}$ will be chosen as entering variable. This means the inequality $A_{s} x \leq b_{s}$ will enter the next station cone $M^{\{k+1\}}$ (if $A_{i} z^{k}=b_{i}$ for some $H_{1}, H_{2}, \ldots, H_{n}, 0$. then we can choose any $\left.i \in\left\{i_{1}, i_{2}, \ldots, i_{k}\right\}\right)$. The point $z^{k}$ will be calculated as follows.

Denote $\bar{I} \subset\{1,2, \ldots, m\}$ such that $A_{i} \quad x^{k}>b i, \mathrm{i}=$ $1,2, \ldots, \mathrm{m}$, we have to find $\lambda_{i}, i \in \bar{I}$ such that $A_{i} z_{i}=b_{i}, i \in \bar{I}$, i.e.

$$
A_{i}\left(\lambda_{i} O+\left(1-\lambda_{i}\right) x^{k}\right)=b_{i}, 0<\lambda_{i}<1, i \in \bar{I} .
$$

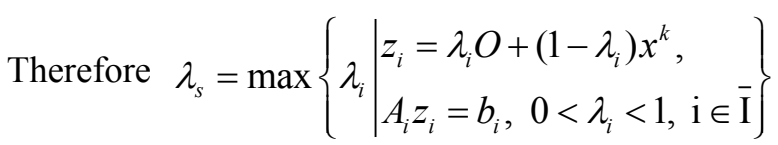
will define the cutting hyperplane $A_{s} x=b_{s}$ and $A_{s}$ is the entering vector into the next station cone $M^{k+1}$. If $A_{i} z^{k}=b_{i}$ for $i \in\left\{i_{1}, i_{2}, \ldots, i_{k}\right\}$ then we can choose any $i \in\left\{i_{1}, i_{2}, \ldots, i_{k}\right\}$.

Now we show that the vertex $x^{k+1}$ of $M^{k+1}$ will produce a smaller value for the objective function.

Theorem 2.5. Let $x^{k}$ be a vertex of $M^{k}$ at step $k$. Suppose $x^{k}$ is a unique optimal solution of $\langle c, x\rangle, \forall x \in M^{k}$. Then

$$
\left\langle c, x^{k+1}\right\rangle\left\langle\left\langle c, x^{k}\right\rangle\right. \text {. }
$$


Proof. Since $x^{k}$ is a unique optimal solution then

$$
\left\langle c, x^{k}\right\rangle>\langle c, x\rangle, \forall x \in M^{k}, x \neq x^{k}
$$

On the other hand

$$
x^{k+1} \in M^{k} .
$$

From (4.6), (4.7), follows $\left\langle c, x^{k+1}\right\rangle<\left\langle c, x^{k}\right\rangle$.

The proof is completed

Remark 1 The assumption $x^{k}$ is a unique optimal solution of $\langle c, x\rangle$ on $M^{k}$ which is equivalent to the assumption that the vector $c$ is a strict positive linear combination of the basis vectors of $M^{k}$ i.e. $\lambda_{k 0}>0, \forall k=1,2, \ldots, n$. This means the dual problem (3.1) of (2.1) is nondegenerated.

\section{Algorithms}

After the above discussion, we now proceed to formulate the following algorithm.

\section{Algorithm 1}

1. Initialization

Determine the starting station cone $M$. Calculate the point $O^{*}$ by formula (4.5).

Let: $M^{k}=M ; O=O^{*}$.

2. Step $(\boldsymbol{k}=\mathbf{1}, \mathbf{2}, \ldots)$

If the vertex $x^{k}$ of the station cone $M^{k}$ is a feasible point of $P$, then $x^{k}$ is an optimal solution. In the contrary case, select the inequality $A_{s} x \leq b_{s}$ for entering the station cone and define the inequality $A_{i_{r}} x \leq b_{i_{r}}$ for leaving the station cone. Determine the new station cone $M^{\{k+1\}}$ with the vertex $x^{\{k+1\}}$.

Go to next step $k=k+1$.

Remark 2 Except for the calculation for finding the entering variable, each step of algorithm 1 is a simplex pivot.

With the assumption that the dual problem (3.1) of (2.1) is nondegenerated, we hence have the following.

\section{Theorem 2.6}

The above algorithm produces an optimal solution after a finite number of iterations.

Proof. Follows from the theorems 2.3,2.4,2.5. It is obvious that the calculation of the interior point $O^{*}$ by formula (4.5) may require additional computational work which influences on the efficiency of the above algorithm. Normally we suggest using the point $O$ instead of $O^{*}$ if $O$ is positioned quite distantly separated from the facets of $P$. We also note that if $O$ is near the optimal solution of (2.1) then the cutting hyperplane can most probably be one of the facets which formulate the optimal solution. Taking this advantage, we can have

$$
O=\frac{1}{2}\left(O+z^{k}\right) \text { where } A_{s} z^{k}=b_{s}
$$

It is clear that point $O$ in the formula (5.1) is a strict interior point which is moving in the direction towards the optimal point step by step. We hope that for some class of linear programming problems the movement of $O$ towards the optimal point can reduce the number of iterations. Therefore we suggest the following:

\section{Algorithm 2}

1. Initialization

Determine the starting station cone $M$. Find the point $O$.

Let $M^{k}=M ; O=O$

2. Step $k(k=1,2, \ldots)$

If the vertex $x^{k}$ of the station cone $M^{k}$ is a feasible point of $P$, then $x^{k}$ is an optimal solution. In the contrary case, select the inequality $A_{s} x \leq b_{s}$ for entering the station cone and define the inequality $A_{i_{r}} x \leq b_{i_{r}}$ for leaving the station cone.

Let $O=\frac{1}{2}\left(O+z^{k}\right)$ where $A_{s} z^{k}=b_{s}$

Determine the new station cone $M^{\{k+1\}}$ with the vertex $x^{\{k+1\}}$.

Go to next step $k=k+1$.

\section{Numerical Example}

Consider the Linear programming problem:

$$
-x_{1}+2 x_{2} \rightarrow \max
$$




$$
\begin{aligned}
& -3 x_{1}+x_{2} \leq 3,-x_{1}+x_{2} \leq 5 \\
& -x_{1}-2 x_{2} \leq-2 \\
& x_{1}+3 x_{2} \leq 36, x_{1}+x_{2} \leq 20 \\
& -x_{1} \leq 0,-x_{2} \leq 3
\end{aligned}
$$

We apply Algorithm 1 to solve problem (6.1).

$$
\text { Initial step: }
$$

\section{- Find an initial station cone}

Find a basis which is a positive linear combination of vector $c$. That means to find a solution of the following system :

$$
\begin{aligned}
& -3 \lambda_{1}-\lambda_{2}-\lambda_{3}+\lambda_{4}+\lambda_{5}-\lambda_{6}=-1 \\
& \lambda_{1}+\lambda_{2}-2 \lambda_{3}+3 \lambda_{4}+\lambda_{5}-\lambda_{7}=2 \\
& \lambda_{j} \geq 0, j=1,2, \ldots, 7, \lambda_{j_{1}}>0, \lambda_{j_{2}}>0, \\
& \lambda_{\mathrm{j}}=0, j \neq j_{1}, j_{2},
\end{aligned}
$$

We use the big - $\mathrm{M}$ augmentation to find a solution of (6.2). It is easy to note that

$$
\begin{aligned}
& \lambda_{5}-\lambda_{6}=-1 \\
& \lambda_{5}=2
\end{aligned}
$$

define a positive linear combination of vector $c$ with $\lambda_{5}=2 ; \lambda_{6}=3$. Then the initial station cone $M^{l}$ will be defined by following system

$$
\begin{aligned}
x_{1}+x_{2} & \leq 20 \\
-x_{1} & \leq 0
\end{aligned}
$$

with vertex $x^{1}=(0,20)$. The vectors $A_{5}=(1,1), A_{6}=(-1,0)$ are basis of $M^{l}$. We note that $x^{1}=(0,20)$ is not a feasible solution of $(6.1)$ then go to step 2 .

- Define a strict initial interior point $O$

Note that $O=(3,3)$ is a strict interior point.

Calculating $O^{*}$

Project $O$ onto the facets of $M^{l}$ (see figure 3), we have: $H_{1}=(9,9) ; H_{2}=(0,3)$. From (3.5) follows

$$
O^{*}=\frac{1}{3}\left(O+H_{1}+H_{2}\right)=(4,5)
$$

$$
\begin{aligned}
&-x_{1}+2 x_{2} \rightarrow \text { max } \\
&-3 x_{1}+x_{2} \leq 3 \\
&-x_{1}+x_{2} \leq 5 \\
&-x_{1}-2 x_{2} \leq-2 \\
& x_{1}+3 x_{2} \leq 36 \\
& x_{1}+x_{2} \leq 20 \\
&-x_{1} \quad \leq 0 \\
&-x_{2} \leq 3
\end{aligned}
$$

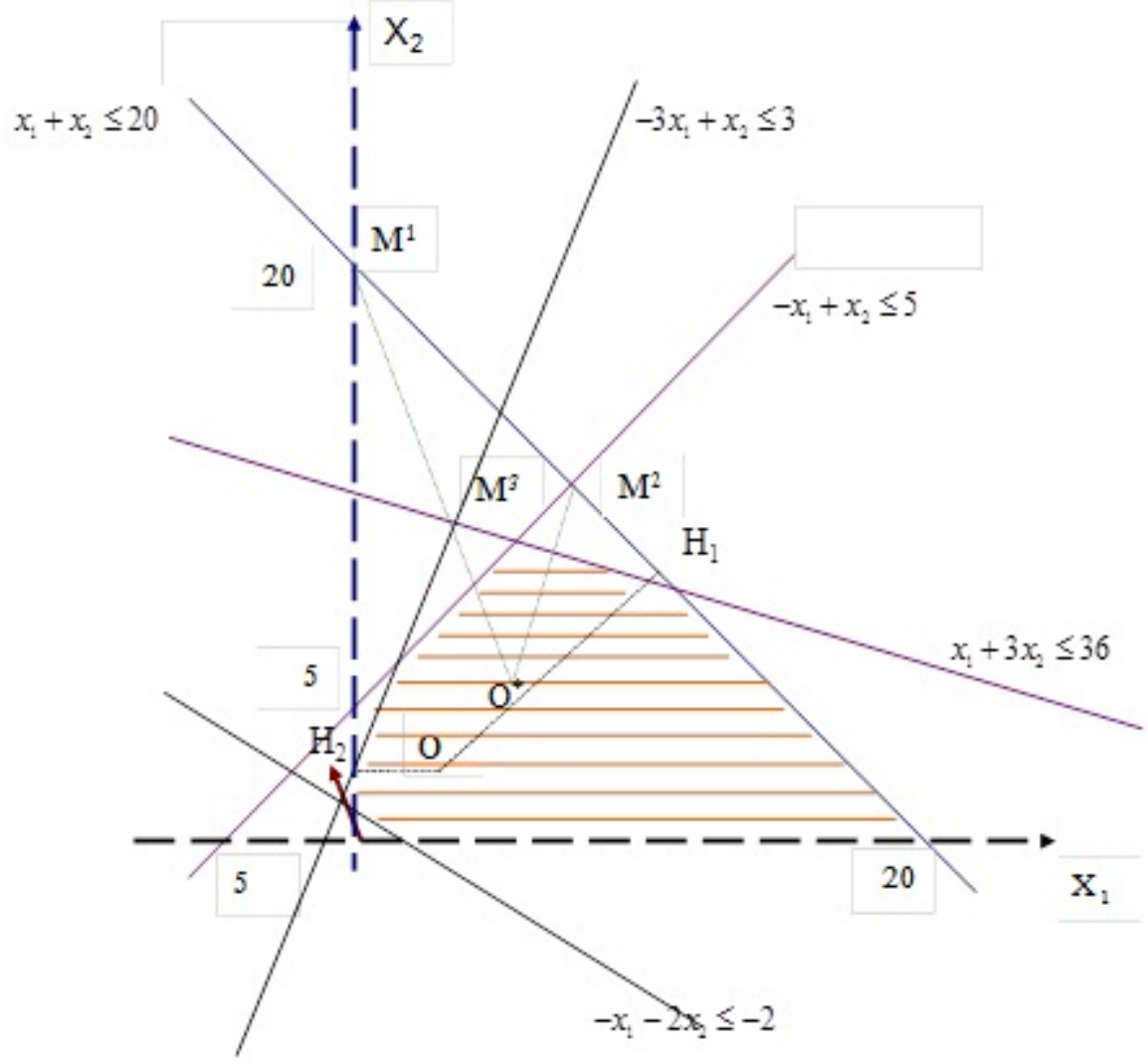

Fig. 3 
Therefore $O=O^{*}=(4,5)$ is a strict initial interior point.

\section{$\underline{\text { Step }} k=1$}

\section{- Finding the cutting hyperplane}

Connect $O=(4,5)$ with $x^{1}=(0,20)$. The segment $\left[O, x^{I}\right]$ intersect with the boundary of $P$ at the inequality $-x_{1}+x_{2} \leq 5$ (see figure 3 ). Therefore $A_{2}=(-1,1)$ is entering vector and the inequality $-x_{1}+x_{2} \leq 5$ is entering into the next station cone.

Denote $\bar{I} \subset\{1,2, \ldots, m\}$ is index set such that $x^{k}$ is violating. Then the cutting hyperplane is defined by

$$
\lambda_{s}=\max \left\{\lambda_{i} \mid \begin{array}{l}
z_{i}=\lambda_{i} O+\left(1-\lambda_{i}\right) x^{k}, \\
A_{i} z_{i}=b_{i}, 0<\lambda_{i}<1, \mathrm{i} \in \overline{\mathrm{I}}
\end{array}\right\}
$$

It is obvious $x^{1}=(0,20)$ is not satisfying the constraints $\quad 1,2,4 \quad(\quad \bar{I}=\{1,2,4\} \quad$. Calculate $\lambda_{1}, \lambda_{2}, \lambda_{4}:$

$$
z_{i}=\lambda_{i} O+\left(1-\lambda_{i}\right) x^{1}=\left(4 \lambda_{i}, 20-15 \lambda_{i}\right) .
$$

Put $Z_{i}$ into the constraints 1, 2, 4. With the constraint 1 we have:

$$
\begin{gathered}
-3 x_{1}+x_{2}=3 \Rightarrow \\
-12 \lambda_{1}+20-15 \lambda_{1}=3 \Rightarrow \\
\lambda_{1}=17 / 27 .
\end{gathered}
$$

With constraint $2: \lambda_{2}=15 / 19$; With constraint $4: \lambda_{4}=24 / 11$.

Then

$$
\lambda_{2}=\max \left\{\lambda_{1}=17 / 27, \lambda_{2}=15 / 19, \lambda_{4}=24 / 11\right\} .
$$

Therefore the inequality $-x_{1}+x_{2} \leq 5$ is entering to the next step.

\section{- Selecting the leaving vector}

Establish the simplex table with basis $A_{5}, A_{6}$ as follows:

\begin{tabular}{|l|l|l|l|l|l|l|l|l|l|}
\hline $\mathrm{N}$ & $\mathrm{Basis}$ & $\mathrm{C}$ & $\mathrm{A}_{1}$ & $\mathrm{~A}_{2}$ & $\mathrm{~A}_{3}$ & $\mathrm{~A}_{4}$ & $\mathrm{~A}_{5}$ & $\mathrm{~A}_{6}$ & $\mathrm{~A}_{7}$ \\
\hline 1 & $\mathrm{~A}_{5}$ & 2 & 1 & 1 & -2 & 3 & 1 & 0 & -1 \\
\hline 2 & $\mathrm{~A}_{6}$ & 3 & 4 & 2 & -1 & 2 & 0 & 1 & -1 \\
\hline
\end{tabular}

(Step 1)

$A_{2}$ is the entering vector and $\lambda_{21}=1>0 ; \lambda_{22}=2>0$;

$\frac{\lambda_{20}}{\lambda_{22}}=\frac{3}{2}=\min \left\{\frac{\lambda_{10}}{\lambda_{12}}=2, \frac{\lambda_{20}}{\lambda_{22}}=\frac{3}{2}\right\}$.

Therefore $A_{6}$ is the leaving vector. The station cone $M^{2}$ with vertex $x^{2}$ defined by system

$$
x_{1}+x_{2} \leq 20 ;-x_{1}+x_{2} \leq 5 \text {. }
$$

Since the vertex $x^{2}=\left(7 \frac{1}{2}, 12 \frac{1}{2}\right)$ does not satisfy

all constraints of (6.1) then we go to next step.

Step $k=2$

- Selecting the cutting hyperplane

Similarly to step $k=1$, we find the inequality $x_{1}+3 x_{2} \leq 36$ is entering to the next step (see figure 3 ).

- Selecting the leaving vector

New simplex table for basis $A_{5}, A_{2}$ :

\begin{tabular}{|l|l|l|l|l|l|l|l|l|l|}
\hline $\mathrm{N}$ & Basis & $\mathrm{C}$ & $\mathrm{A}_{1}$ & $\mathrm{~A}_{2}$ & $\mathrm{~A}_{3}$ & $\mathrm{~A}_{4}$ & $\mathrm{~A}_{5}$ & $\mathrm{~A}_{6}$ & $\mathrm{~A}_{7}$ \\
\hline 1 & $\mathrm{~A}_{5}$ & $1 / 2$ & -1 & 0 & $-3 / 2$ & 2 & 1 & $-1 / 2$ & $-1 / 2$ \\
\hline 2 & $\mathrm{~A}_{2}$ & $3 / 2$ & 2 & 1 & $-1 / 2$ & 1 & 0 & $1 / 2$ & $-1 / 2$ \\
\hline
\end{tabular}

(step 2)

Similarly we have $A_{4}$ is the entering vector and $A_{5}$ is the leaving vector. The station cone $M^{3}$ and the vertex $x^{3}$ are defined by

$$
x_{1}+3 x_{2} \leq 36 ;-x_{1}+x_{2} \leq 5 \text {. }
$$

The vertex $x^{3}=\left(5 \frac{1}{4}, 10 \frac{1}{4}\right) \quad$ satisfies all 
constraints of (6.1). Then $x^{3}$ is an optimal solution of (6.1) and $\left\langle c, x^{3}\right\rangle=15 \frac{1}{4}$.

\section{Computational Experiences}

The proposed Algorithm 1 has been tested, using MatLab, on a set of randomly generated linear problems [13] of the form

$$
\left\{\begin{array}{c}
\max \langle c, x\rangle \\
A x \leq b,
\end{array}\right.
$$

Where $c=(1,1, \ldots, 1) \in R^{n}, A$ is the full matrix of $(n \times m)$ with $a_{i j}$ is randomly generated from the interval $[0,1)$, the vector $b$ has been chosen such that the hyperplanes $\left\langle A_{i}, x\right\rangle=b_{i}, i=1, \ldots, m \quad$ are tangent to the sphere $(0,1)$ with center at origin and radius $r=1$. To ensure that (7.1) has a finite optimal solution we add the constraints

$$
x_{i} \leq 1, \quad i=1,2, \ldots, n .
$$

The optimal solution and objective function value of ((7.1)-(7.2)) have been retested by simplex algorithm from MatLab.

Function Data01. m randomly generates the input data for the problems and stores the matrix $A$ and, vector $b$ in the data base form Dat01. mat. Function Alg01. $\mathrm{m}$ solves the problem by a new proposed algorithm1 and function Simplex01. $m$ itself isthe simplex algorithm from the optimization toolbox of MatLab.

We would like to remark that, in order to find the next vertex of station cone, instead of calculating a simplex table, here we call procedure from MatLab to solve the system of linear equalities $A x=b$. This will take much more computing time, especially when $n$ and $\mathrm{m}$ are large numbers. We have tested several

\begin{tabular}{|c|c|c|c|c|c|}
\hline \multirow{2}{*}{$\mathrm{n}$} & \multirow{2}{*}{$\mathrm{m}$} & \multirow{2}{*}{ Problem } & \multicolumn{3}{|l|}{ Iterations } \\
\hline & & & PHASE II SIMPLEX & DUAL SIMPLEX & INEX \\
\hline \multirow{3}{*}{40} & \multirow{3}{*}{200} & 1 & 1529 & 1300 & 216 \\
\hline & & 2 & 1438 & 1600 & 234 \\
\hline & & 3 & 1438 & 1390 & 229 \\
\hline \multirow{3}{*}{100} & \multirow{3}{*}{200} & 1 & 5489 & 7443 & 830 \\
\hline & & 2 & 6715 & 8528 & 865 \\
\hline & & 3 & 6351 & 8293 & 882 \\
\hline 300 & 1000 & 1 & 212261 & 227215 & 8952 \\
\hline 400 & 1000 & 2 & & 388676 & 13266 \\
\hline 500 & 1000 & 3 & & 583464 & 21033 \\
\hline
\end{tabular}
hundreds examples. Some of them as follows:

\section{Conclusions}

The above tested examples show that the number of iterations of the interior exterior approach is significantly smaller than that of the second phase of the simplex method. Additionally, when the number of variables and constraints of the problem increase, the number of iterations of the interior exterior approach increase in a slower manner than that of the simplex method. In order to gain a more precise conclusion on the effectiveness of the above proposed algorithm 1, there is a need to re-program Algorithm 1 and then carry out computational experiments on a larger scale together with a bigger number of variables and constraints.

\section{References}

[1] M.L. Balinski, Mathematical programming: journal, society, recollections, in: J.-K. Lenstra, A. H. G. Rinnooy Kan et A. Schrijver, eds., History of Mathematical Programming: a Collection of Personal Reminiscences, CWI et North-Holland Publishing Company, Amsterdam, pp. 5-18.

[2] R.E. Bixby, Implementing the simplex method: The initial basis, ORSA Journal on Computing 4 (1992) 267-284.

[3] R.E.Bixby, Progress in linear programming, ORSA 
Journal on Computing 6(1) (1994) 15-22.

[4] S.N. Chernikov, Linear Inequalities, Nauka, Moscow, 1968 (in Russian).

[5] G.B. Dantzig, Maximization of a linear function of variables subject to linear inequalities, In Koopmans, T.C., ed., Activity Analysis of Production and Allocation, Wiley, New York, 1951, pp. 339-347.

[6] G.B. Dantzig, Progress in linear programming, ORSA Journal on Computing 6(1) (1963) 15-22.

[7] J.J. Forrest, D. Goldfarb, Steepest-edge simplex algorithms for linear programming, Mathematical Programming 57 (1992) 341-374.

[8] N.K. Karmarkar, A new polynomial-time algorithm for linear program-ming, Combinatorica 4 (1984) 373-395.

[9] L.G. Khachiyan, A polynomial algorithm in linear programming (in Russian), Doklady Akademiia Nauk SSSR 224 (1979) 1093-1096. English translation: Soviet Mathematics Doklady, 20, 191-194.

[10] V. Klee, G. J. Minty, How good is the simplex algorithm?,
In: Shisha, O., ed., Inequalities, III, Academic Press, 1972, pp. 159-175. iterations. J. Res. Nat. Bur. Stand 49 (1952) 33-53.

[11] K. G. Murty, A Gravitational Interior Point Method for LP, Opsearch 42(1) (2005) 28-36.

[12] K. G. Murty, A New Practically Efficient Interior Point Method for LP, Algorithmic Operations Research 1 (2006) 3-19.

[13] K. Paparrizos *, N. Samaras, G. Stephanides, An efficient simplex type algorithm for sparse and dense linear programs, European Journal of Operational Research 148 (2003) 323-334.

[14] S. Smale, On the average number of the simplex method of linear programming, Mathematical Programming 27 (1983) 241-262.

[15] M.J. Todd, The many facets of linear programming, Mathematical Programming 91 (2002) 417- 436.

[16] Y. Ye, Interior Point Algorithm, Theory and Analysis, Wiley-Interscience, 1997. 